Supplement of Hydrol. Earth Syst. Sci., 24, 2873-2894, 2020

https://doi.org/10.5194/hess-24-2873-2020-supplement

(c) Author(s) 2020. This work is distributed under

the Creative Commons Attribution 4.0 License.

(c) (1)

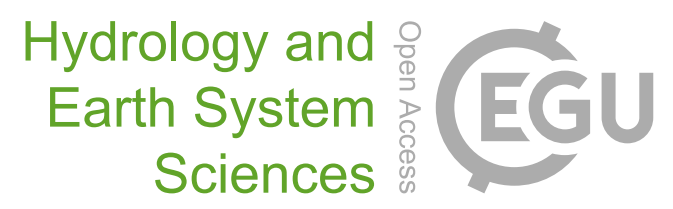

Supplement of

\title{
Linking economic and social factors to peak flows in an agricultural watershed using socio-hydrologic modeling
}

David Dziubanski et al.

Correspondence to: David Dziubanski (dave.dziubanski@gmail.com)

The copyright of individual parts of the supplement might differ from the CC BY 4.0 License. 


\section{S1.1 Stochastic Variability of Agricultural Economic Variables}

Differences in land quality causes production costs to range by approximately $20 \%$ between low and high quality land, with $50-60 \%$ of the difference due to cash rental rates, and $30-40 \%$ of the difference due to crop production inputs (i.e. seed, fertilizers, crop insurance) (Plastina, 2017b). To account for the impact of land quality, crop production costs are adjusted and stochastically varied using values and ranges derived from historical crop production costs for 2000-2018 and Cash Rental Rates from Iowa surveys for 2002-2017 (Plastina, 2017b, 2017a).

The production costs of a farmer agent are adjusted up or down based on the average Corn Suitability Rating (CSR2) value of the agent's land. CSR2 values for different soil and field conditions available from the NRCS SSURGO database were used to calculate a weighted average CSR2 value according to the fraction of soil types present (Section S2). Based on the 2015-2017 Iowa State University Land Value surveys (Zhang, 2017), low, medium, and high quality CSR2 values were determined to be 61,74 , and 86, respectively. Assuming that the historical average production costs correspond to medium quality land, a farmer agent's production costs are scaled linearly using a 10\% decrease in the median cost for the lowest quality land and a $10 \%$ increase in the median cost for the highest quality land.

Stochastic variability was added to the production costs based on variability in cash rent and crop production input prices. Cash rent variability was found to be consistent across land qualities, but changed with crop price. The $25^{\text {th }}$ percentile of cash rent linearly decreased from $10 \%$ to $-40 \%$ from the average value between a crop price of $\$ 2.00$ and $\$ 7.00$, and was represented through the regression equation: 


$$
\text { Decrease }=-0.0449 \times \text { CropPrice }-0.0982
$$

The $75^{\text {th }}$ percentile of cash rent linearly increased from $+10 \%$ to $+45 \%$ from the average value between a crop price of $\$ 2.00$ and $\$ 7.00$, and was represented through the regression equation:

$$
\text { Increase }=0.0565 \times \text { CropPrice }+0.0780
$$

Based on the crop price, the stochastic variability added to the cash rent is randomly drawn from the uniform distribution: $\mathcal{U}($ Decrease, Increase). Further, it is assumed that cash rents are unlikely to vary significantly from below to above the mean cash rent from one year to the next. Therefore, the final variability added to the cash rent is based on a weighted average between the previous year's and current year's variability, with equal weight place on both years.

The inputs portion of the production costs (i.e. cost of seed, fertilizer, crop insurance, etc.) increased and decreased by $\sim 3-4 \%$ of the total historical production costs between medium and high quality land, and medium and low quality land, respectively. It was assumed that variability in the input costs could range between $+1.5 \%$ and $-1.5 \%$ of the total production costs. Thus, input variability was randomly drawn from a uniform distribution: $\mathcal{U}(-0.015,0.015)$. The final production cost is represented by the equation:

$$
\begin{aligned}
\text { ProdCost }_{t} & =\left[\text { ProdCostHist }_{t} \cdot \text { ProdCostAdjust }_{(\text {CSR2 })]}\right. \\
& \left.+ \text { CashRentRandom }_{(\text {CropPrice }}\right) \\
+ & {\left[\text { ProdCostHist }_{t} \cdot \text { InputCostRandom }\right] }
\end{aligned}
$$

where ProdCost $_{t}$ is the final adjusted production cost for year $t$, ProdCostHist $t_{t}$ is the historical production costs, ProdCostAdjust(CSR2) is the production cost adjustment based on CSR2 value, CashRentRandom (CropPrice ${ }_{t}$ ) is the stochastic variability added to cash rent based on Eqs. (S1) and (S2), and InputCostRandom is stochastic variability added to input costs. 


\section{S1.2 Opportunity Cost Adjustment}

Because production costs vary based on land quality, opportunity costs are adjusted up or down such that farmer agents with higher (lower) land quality will have higher (lower) opportunity costs associated with implementing conservation land.

Based on financial analysis conducted by (Tyndall et al., 2013), farmer agents consider three key cost variables associated with implementing conservation land: cost of establishing the prairie (i.e. materials, site preparation and planting), cost of maintaining the prairie through annual mowing or burning, and cost of forgone revenue from the land taken out of crop production. Opportunity costs account for $53 \%$ of total costs of native prairie strips for low quality land and scale up to $88 \%$ for high quality land, while establishment costs account for $10 \%$ or less of total costs for high quality land and scale up as land quality decreases (Tyndall et al., 2013). For simplicity purposes, we assume opportunity costs are $90 \%$ of $\operatorname{Cost}_{\text {prairie }}$ under high quality land and $60 \%$ of Cost $_{\text {prairie }}$ under low quality land. The remaining percentage of Cost $_{\text {prairie }}$ is assumed to be split evenly between establishment and maintenance costs. The final opportunity cost for farmer agents with land between low and medium quality is calculated as:

$$
\text { OppCost }=\text { OppCostIncreasedPerCSR2 } \cdot \text { WeightedAvgCSR }-0.104
$$

where OppCostIncreasedPerCSR2 is the increase in opportunity costs per point increase in the CSR2 value (i.e. slope), and the value -0.104 is the intercept which gives an opportunity cost of $60 \%$ of total costs at a CSR2 value of 61 . A similar equation is used for finding the opportunity cost between medium and high quality land, with the difference being the intercept coefficient. The final cost per acre of implementing conservation land is calculated using the following equation:

$$
\text { Cost }_{\text {prairie:t }}=\text { OppCost }+ \text { Establishment }+ \text { Maintenance }
$$


where OppCost is based on the cash rent input, and establishment and maintenance costs are calculated as:

$$
\frac{\text { Establishment }}{\text { Maintenance }}=\text { CashRent } \cdot \frac{\frac{1-0.9}{2}}{0.9}
$$

\section{S2. Soil Crop Yield Adjustment and Stochastic Variability}

A farmer agent's land can be defined by up to 8 different soil types at different percentages. The soil types used are common to the Squaw Creek watershed: Nicollet Loam (1$3 \%$ slope), Clarion Loam (2-5\% slope), Webster clay loam (0-2\% slopes), Canisteo clay loam (02\% slopes), Clarion loam (5-9\% slopes, moderately eroded), Harps loam (1-3\% slopes), Clarion loam (5-9\% slopes), and Okoboji mucky silt loam (0-1\% slopes). To account for differences in soil crop productivity, adjustments were developed using field scale data spanning 1995-2006 from 10 fields in central Iowa (USDA-ARS Cooperative Grower 3625-13660-14S). Mean yearly yields were derived for each soil type over the course of the 11-year period. The mean yearly yields for each soil type were compared against mean historical central Iowa crop yields for the same period. Soil type adjustments were calculated as the mean differences between the mean yearly yield and mean historical yield. In general, the soils displayed on average a $0.35 \mathrm{Mt} / \mathrm{Ha}$ higher yield over mean historical central Iowa yields. For Clarion loam (5-9\% slopes, moderately eroded), yields were approximately $0.11 \mathrm{MT} / \mathrm{Ha}$ lower than the mean central Iowa yield, while Nicollet Loam (1-3\% slope) on average produced 0.63 MT/Ha higher yields. All other soil type displayed differences in mean yield between these values. The adjustment factors were added to the crop yield for each soil type over the entire simulation period, thereby assuming that the factors are constant through time. 
To account for other factors that influence crop productivity, stochastic variability drawn from a uniform distribution between the $5^{\text {th }}$ and $95^{\text {th }}$ percentile yields for each soil type are added to the yield for that year. For example, the $5^{\text {th }}$ and $95^{\text {th }}$ percentiles of yields for Nicollet Loam were on average $0.9 \mathrm{MT} / \mathrm{Ha}$ lower and $0.95 \mathrm{Mt} / \mathrm{Ha}$ higher than the mean yearly yield for Nicollet loam. Thus, after adjusting the soil yield up by $0.63 \mathrm{MT} / \mathrm{Ha}$, stochastic variability was then added from the uniform distribution: $\mathcal{U}(-0.9,0.95)$.

\section{S3. Farm Agent Network}

The farmer agent network influences the diffusion of conservation adoption. Farmers trust their neighbors for providing reliable information about crop production (Arbuckle, 2017) and are heavily influenced by their neighbors' practices (Davis and Gillespie, 2007; McGuire et al., 2013; Saltiel et al., 1994). In the farmer agent module, a probabilistic-based network is established where the number of connections that an agent makes is randomly drawn from a binomial distribution (Newman et al., 2002) which describes the probability of forming $k$ connections:

$$
P(k)=\left(\begin{array}{c}
n-1 \\
k
\end{array}\right) p^{k}(1-p)^{n-1-k} \quad n \in\{0, \ldots, n-1\}
$$

where a farmer in a subbasin of $n$ agents can make up to $n-1$ connections, each with the same success probability of $p$. Currently, $p$ is set to 0.5 in the model, indicating a $50 \%$ probability of forming a connection with any one farmer. A second parameter that describes the farmer network is the connection strength, ConnStrength (Granovetter, 1973) which indicates the probability of the agents sharing their land use information during any given year. Once a farmer agent initiates a connection with another farmer agent, their connection strength is randomly chosen from the uniform distribution: $\mathcal{U}(0,1)$. A farmer agent wanting to communicate with 
another farmer agent is defined by a random choice from a Bernoulli distribution with $p=$ ConnStrength. If the choice of connection is a success for both farmer agents, they share information; however, if the choice of connection is a success for only one farmer agent (i.e. one farmer agent wants to communicate with the other, but the other farmer agent does not want to communicate back), then the agents do not share information.

\section{S4. Farmer Agent Profit Decisions}

The past profits decision is solely based on outcomes that have been fully realized for the previous $X$ years. In this decision, the land allocated to conservation is based on the net amount of money that could have been earned per hectare of conservation land versus crop land and is calculated as:

$$
\delta C_{\text {profit: } X}=\left[A * \text { Profit }_{\text {diff }}{ }^{2}+B * \text { Profit }_{\text {diff }}+C\right] \cdot \text { Cons }_{\text {max }} \cdot \text { Hectares }_{\text {tot }}
$$

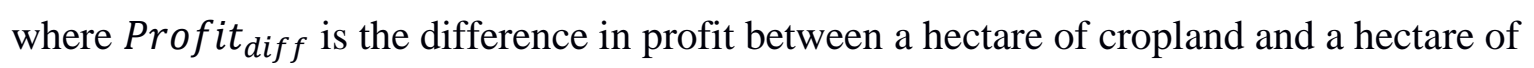
conservation land (Table S1), Cons $\max _{\max }$ is the farmer agent's maximum conservation parameter, Hectacres $_{\text {tot }}$ is the area of the agent's land, and $A, B, C$ are equation coefficients discussed later.

Profit $_{\text {diff }}$ is calculated as:

where,

$$
\text { Profit }_{\text {diff }}=\frac{1}{n} \sum_{t=t-1}^{t=t-X} \text { Profit } \text { crop:t }- \text { Profit }_{\text {cons: }}
$$

and,

$$
\text { Profit }_{\text {crop:t }}=\left(\text { CropPrice }_{t} \cdot \text { Yield }_{t}\right)-\text { ProdCost }_{t}+\text { FedSub }_{t}+\text { CropIns }_{t}
$$

$$
\text { Profit }_{\text {cons:t }}=\text { ConsSubsidy }_{t}-\text { Cost }_{\text {prairie: }}
$$


Profit $_{\text {crop:t }}$ is the profit received for cropland in year $t$ (Table S1), CropPrice $_{t}$ is the realized crop price for year $t$, Yield $t_{t}$ is the farmer's realized mean yield (per hectare) for year $t$, ProdCost $_{t}$ is the crop production cost for year $t, F e d S u b_{t}$ is the amount of federal subsidies the farmer received in year $t$, CropIns $_{t}$ is the total amount of crop insurance received in year $t$ (Section S6), Profit cons:t $_{t}$ is the profit received for conservation land in year $t$, ConsSubsidy $y_{t}$ is the conservation subsidy established in year $t$, and Cost $_{\text {prairie:t }}$ is the cost associated with establishing and maintaining conservation land in year $t$ (Section S1.2).

The future crop prices decision is based on a combination of past performance information and projected future crop prices. Equations (S8), (S9) and (S11) are used to calculate the land allocated to conservation based on future crop price, $\delta C_{f u t u r e s: Y}$, with CropPrice $_{t}$ in Eq. (S10) being replaced with CropPrice $_{t: t+Y}$ CropPrice $_{t: t+Y}$ is the projected crop price for the $Y$ upcoming growing seasons, and is based on historical crop prices with an added adjustment calculated from historical errors in crop price forecasts produced by the U.S. Department of Agriculture (Section S5).

The first term in Eq. (S8) is a second-degree polynomial of form $A x^{2}+B x+C=y$, therefore three equations need to be simultaneously solved to determine coefficients $A, B, C$. $\delta C_{\text {profit }}$ and $\delta C_{\text {futures }}$ can take on values between $-100 \%$ to $100 \%$ depending upon whether the

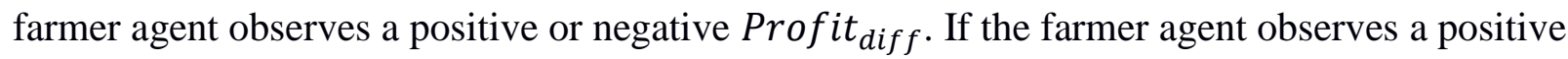
Profit $_{\text {diff }}$, the agent uses all historical positive Profit diff values from the start of the simulation through $t-1$ to solve for the coefficients using the following system of equations: 


$$
\begin{gathered}
A\left(P_{\text {upper }}\right)^{2}+B\left(P_{\text {upper }}\right)+C=- \text { maxChange } \\
A\left(P_{\text {middle }}\right)^{2}+B\left(P_{\text {middle }}\right)+C=-0.5 \text { maxChange } \\
A\left(P_{\text {lower }}\right)^{2}+B\left(P_{\text {lower }}\right)+C=0
\end{gathered}
$$

where $P_{\text {upper }}, P_{\text {middle }}$, and $P_{\text {lower }}$ are upper, middle, and lower percentiles of the historical

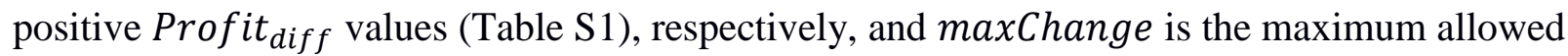
change in conservation land in any given year, which is equal to 1.0 (up to $100 \%$ change possible). In this study, $P_{\text {upper }}, P_{\text {middle }}$, and $P_{\text {lower }}$ are set to the $75^{\text {th }}, 50^{\text {th }}$, and $25^{\text {th }}$ percentiles. When Profit diff is positive (i.e. greater profit was earned from crop production than conservation land), the farmer agent will potentially decrease the amount of land in conservation. A similar process occurs when the farmer observes a negative Prof it $_{\text {diff }}$; however, the system of equations now becomes:

$$
\begin{gathered}
A\left(P_{\text {lower }}\right)^{2}+B\left(P_{\text {lower }}\right)+C=\max C h a n g e \\
A\left(P_{\text {middle }}\right)^{2}+B\left(P_{\text {middle }}\right)+C=0.5 \text { maxChange } \\
A\left(P_{\text {upper }}\right)^{2}+B\left(P_{\text {upper }}\right)+C=0
\end{gathered}
$$

Under negative Profit ${ }_{\text {diff }}$, conservation land is potentially increased because the farmer earned a lower revenue through crop production. 


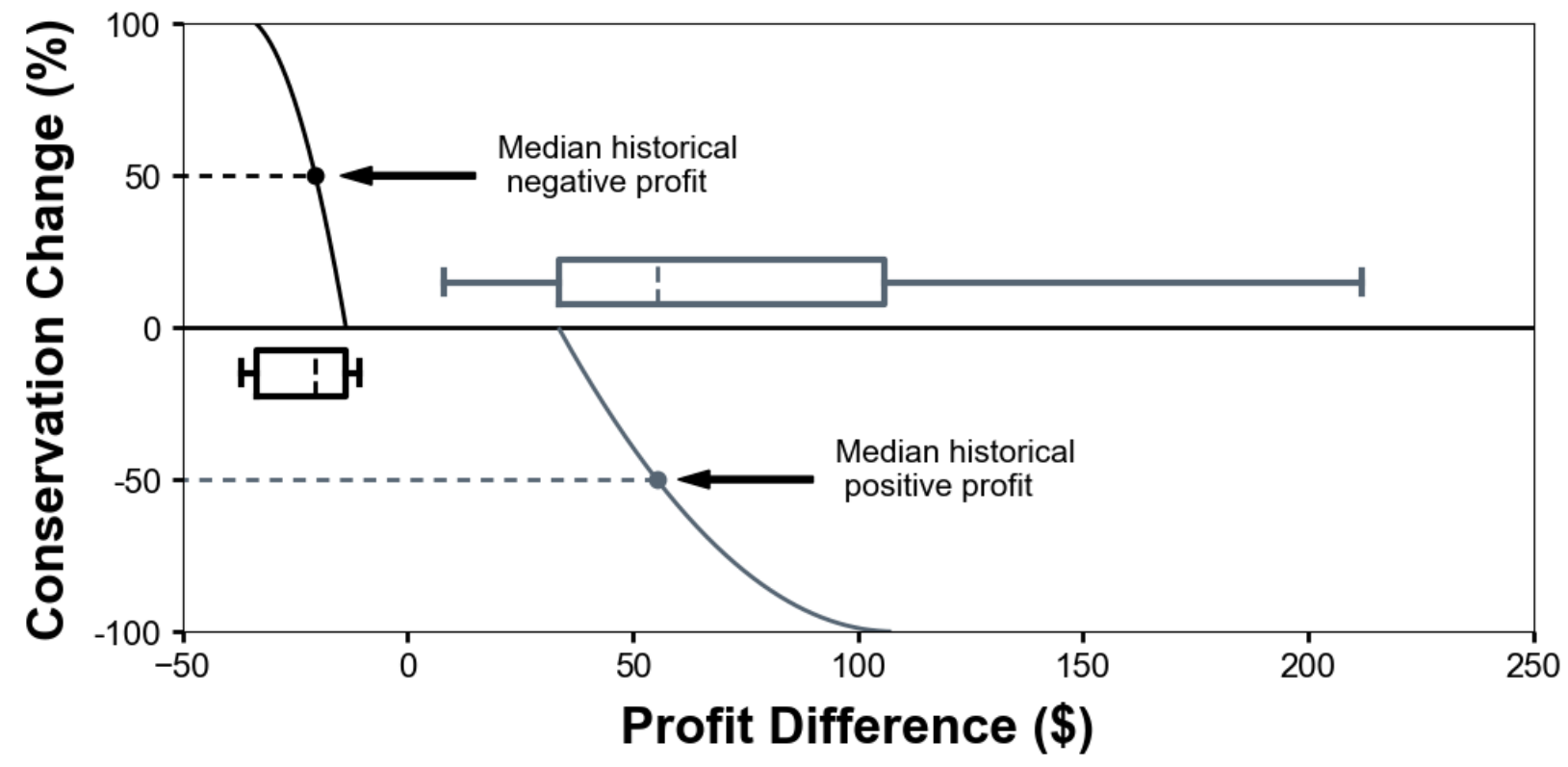

Figure S1. Example of percent conservation change for $\delta C_{\text {profit }}$ and $\delta C_{\text {futures }}$. Gray curves indicate negative percent change (decrease conservation land), black curves indicate positive percent change (increase conservation land).

Equations (S12) and (S13) are illustrated in Fig. S1. Half of the maximum allowable percent increase in conservation land $(+0.5$ maxChange $)$ is assumed to correspond to the

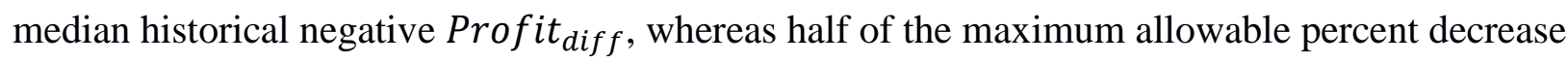
in conservation land ( -0.5 maxChange) corresponds to the median historical positive Profit $_{\text {diff }}$ (Figure S1). We assume that farmer agents will not change land use when a very small profit difference between the two possible options is observed because changing land use requires extra upfront time and resources (Duffy, 2015). Similarly, we assume that farmer agents will fully implement the maximum land conversion possible prior to reaching the most extreme Profit $_{\text {diff }}$ values. Therefore, an increase in conservation land begins to occur when the negative Profit $_{\text {diff }}$ value is less than the $75^{\text {th }}$ percentile of historical negative Profit ${ }_{\text {diff }}$ and reaches a maximum (100\% change) when the negative Profit $t_{\text {diff }}$ reaches the $25^{\text {th }}$ percentile (black curve 
in Figure S1). A decrease in conservation land begins to occur when the positive Profit diff $_{f}$ is greater than the $25^{\text {th }}$ percentile of historical positive Profit ${ }_{\text {diff }}$ and reaches a maximum (-100\%) when the positive Profit ${ }_{\text {diff }}$ reaches the $75^{\text {th }}$ percentile (gray curve in Figure S1). A farmer

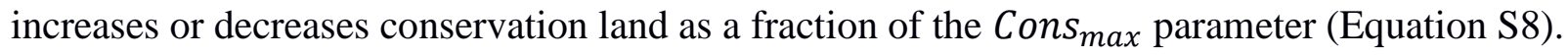

\section{S5. Market Module}

The primary purpose of the market is to provide forecasts of crop prices at the start (February) of each year and realized crop prices at the end (October) of each year when farmer agents harvest their crop. Each farmer agent receives yearly forecasts of future crop prices that predict crop prices for 10 years into the future (i.e. year $t$ to year $t+10$ ). The market module formulates a forecast based on historical crop prices and error estimates of U.S Department of Agriculture (USDA) crop price forecasts.

Twelve years of USDA crop price forecasts for 2001-2012 were analyzed against realized crop prices to form error functions. For each 10-year forecast, errors were calculated between the historical crop prices and the forecasted crop prices. Through marketing year 2009/2010, errors were generally in the $-\$ 1 / \mathrm{Bu}$ to $-\$ 2 / \mathrm{Bu}$ range $(-\$ 39.3 / \mathrm{MT}$ to $-\$ 78.7 / \mathrm{MT})$ with errors reaching a peak of $-\$ 4.44 / \mathrm{Bu}(-\$ 174.5 / \mathrm{MT})$ for 2012/2013 when crop prices were high. A multiple linear regression was performed between crop price magnitude, time from forecast year and error. In general, crop price magnitude explained most of the error pattern in each forecast, with error showing little correspondence with time from forecast year. When the starting crop price was low (i.e. crop price at the beginning of the forecast), the USDA forecasts underestimated future high crop prices by as much as 50-60\% (Figure S2, errors labeled <2007). However, when starting crop prices were higher (Figure S2, errors labeled > 2007), the USDA forecasts underestimated future high crop prices by $20-40 \%$. From $\$ 2 / \mathrm{Bu}-\$ 7 / \mathrm{Bu}(\$ 78.7 / \mathrm{MT}-$ 
$\$ 275.1 / \mathrm{MT})$, errors change from approximately $+30 \%$ to $-40 \%$. The error for each forecast was modeled as:

$$
\text { Error }_{\text {CropPrice }}=A \cdot \text { Price }^{2}+B \cdot \text { Price }+C
$$

where A, B, C are coefficients from the regression. For each year from 2001-2011, the Pearson's r value was 0.9 or higher except for 2012, which had a Pearson's $r$ of 0.71 . This is indicative of a strong relationship between price and error.

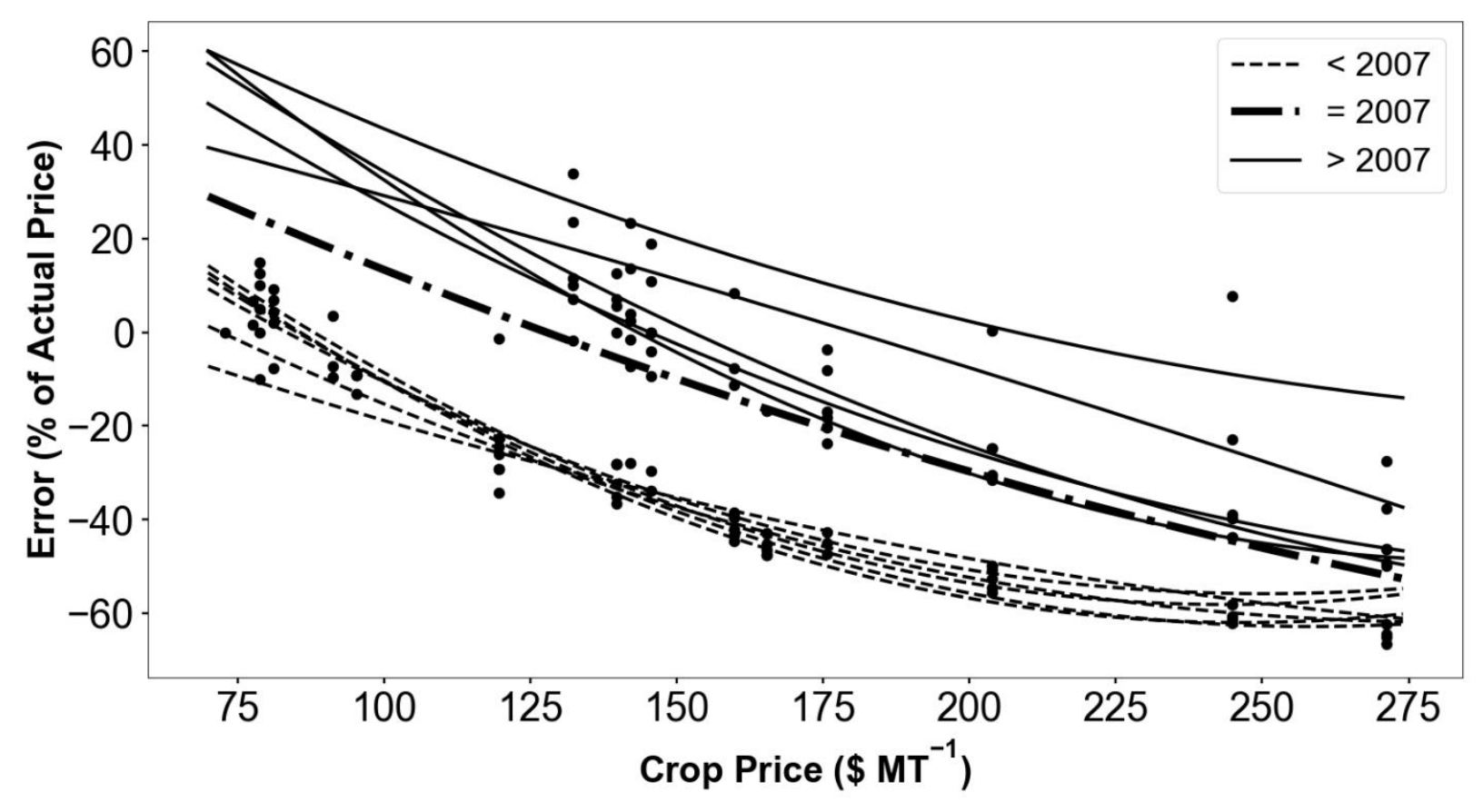

Figure S2. Relationships between crop price and error used in the Market Agent module. Fine dotted curves indicate errors for forecasts prior to 2007 while solid curves indicate errors for forecasts after 2007.

In the market module, each of the 12 error equations for the 12 price forecasts are used (Figure S2) and the error equation with a starting crop price closest to the current year's crop price is chosen for formulating the 10-year forecast based using the following equation:

$$
\text { CropPriceForecast }_{t+n}=\text { CropPrice }_{t+n}+\left(\frac{\text { Error }_{\text {CropPrice }}}{100} \cdot \text { CropPrice }_{t+n}\right)
$$


where CropPriceForecast $t_{t+n}$ is the forecasted crop price for year $t+n$ ( $t$ is the current year), CropPrice $_{t+n}$ is the historical crop price for year $t+n$, and Error $_{\text {CropPrice }}$ is the error based on CropPrice $_{t+n}$.

\section{S6. Farmer Agent Crop Insurance}

The crop insurance protection in the model follows the Revenue Protection (RP) plan, which accounts for $95-100 \%$ of the insurance plans chosen by farmers in the U.S corn belt (Schnitkey, 2017) from the options available through the USDA Agricultural Risk Management agency in the Common Crop Insurance policy. Each farmer agent is on the RP crop insurance plan at an $80 \%$ coverage level with insurance premiums factored into the crop production costs. The RP plan protects farmer agents against poor yields and suppressed crop prices. In a given year, the insurance payout per hectare (Gross Indemnity) at an 80\% coverage level for corn crop is calculated as (Plastina, 2014a):

$$
\text { Gross Indemnity = Rev Guarantee }- \text { Actual Rev }
$$

where,

and,

$$
\begin{gathered}
\text { Rev Guarantee }=\max (\text { Projected Price, } \text { Harvest Price }) * \text { Coverage Level } \\
* \text { TrendAdj APH Yield }
\end{gathered}
$$

$$
\text { Actual Rev }=\text { Harvest Price } * \text { Harvest Yield }
$$

The trend-adjusted actual production history (APH) yield is calculated as (Plastina, 2014b):

$$
\text { TrendAdj APH Yield }=\frac{\sum_{n=1}^{n=10} \text { Yield }_{t-n}+\text { AdjustmentFactor } \cdot n}{10}
$$

where Yield $_{t-n}$ is the historical yield for year $t-n$, with year $t$ being the current year. The yield is based on the mean yield of the previous 10 years. For each year, the yield is adjusted upward by AdjustmentFactor $\cdot n$, where $n$ is the number of years back in time from the current year. The most recent adjustment factors for Iowa have ranged from 1.90 -2.40 (Plastina, 2014b), with 
an AdjustmentFactor value of 2.0 used in the model. The mean of the adjusted yields then gives the trend-adjusted APH yield.

\section{S7. Flood Damage Calculation}

Flood damages are calculated using a flood damage function defined by Tesfatsion et al. (2017):

$$
F D a m=\frac{F D \max }{1+\exp [-(\operatorname{peak} Q-Q 50) / d Q)]}
$$

where FDam is the total flood damage in dollars (\$) (Table S2), FDmax is the maximum flood damage that can be incurred in dollars (\$), Q50 is the flow at which damage is $50 \%$ of the maximum, and $d Q$ is the width of the transition of the flood damage curve. $Q 50$ is defined as:

$$
Q 50=\frac{Q_{\min }+Q_{\max }}{2.0}
$$

where $Q_{\min }$ is the flow at which damage is $1 \%$ of the maximum damage and $Q_{\max }$ is the flow at which damage is $99 \%$ of the maximum damage. Currently, $Q_{\min }$ is set to $317.46 \mathrm{~m}^{3} / \mathrm{s}$ and $Q_{\max }$ is set to $620.62 \mathrm{~m}^{3} / \mathrm{s}$. These values were obtained by simulating the Squaw Creek Watershed at Ames, IA over the 1970-2016 period and calculating the 10 year flood stage and 100 year flood stage. Maximum damage is set to $\$ 50000000$ based on estimates of flood damage during the 2010 Ames, IA flood. $d Q$ specifies how rapidly flood damages accrue from minor flood stage to maximum flood stage (Tesfatsion et al., 2017):

$$
d Q=\frac{Q_{\max }-Q_{\min }}{9.2}
$$




\section{References}

Arbuckle, J. G.: Iowa Farm and Rural Life Poll 2016 Summary Report, Ames, IA., 2017.

Davis, C. G. and Gillespie, J. M.: Factors affecting the selection of business arrangements by U.S. hog farmers, Rev. Agric. Econ., 29(2), 331-348, doi:10.1111/j.1467-9353.2007.00346.x, 2007.

Duffy, M.: Conservation Practices for Landlords, Ames, IA., 2015.

Granovetter, M.: The Strength of Weak Ties, Am. J. Sociol., 78(6), 1360-1380, 1973.

McGuire, J., Morton, L. W. and Cast, A. D.: Reconstructing the good farmer identity: Shifts in farmer identities and farm management practices to improve water quality, Agric. Human Values, 30(1), 57-69, doi:10.1007/s10460-012-9381-y, 2013.

Newman, M. E. J., Watts, D. . J. and Strogatz, S. H.: Random graph models of social networks, Pnas, 99(suppl. 1), 2566-72, doi:10.1073/pnas.012582999, 2002.

Plastina, A.: Current Crop Insurance Policies, Ames, IA., 2014a.

Plastina, A.: Trend-Adjusted Actual Production History (APH), Ames, IA., 2014b.

Plastina, A.: Cash Rental Rates for Iowa 2017 Survey, Ames, IA., 2017a.

Plastina, A.: Estimated Costs of Crop Production in Iowa - 2017, Ames, IA., 2017b.

Saltiel, J., Bauder, J. W. and Palakovich, S.: Adoption of Sustainable Agricultural Practices:

Diffusion, Farm Structure, and Profitability, Rural Sociol., 59(2), 333-349, 1994.

Schnitkey, G.: Revenue Protection (RP) Use on Corn in the Midwest, Champaign, Il., 2017.

Tesfatsion, L., Rehmann, C. R., Cardoso, D. S., Jie, Y. and Gutowski, W. J.: An agent-based platform for the study of watersheds as coupled natural and human systems, Environ. Model. Softw., 89, 40-60, doi:10.1016/j.envsoft.2016.11.021, 2017.

Tyndall, J. C., Schulte, L. A., Liebman, M. and Helmers, M.: Field-level financial assessment of 
contour prairie strips for enhancement of environmental quality, Environ. Manage., 52(3), 736747, doi:10.1007/s00267-013-0106-9, 2013.

Zhang, W.: 2017 Iowa State University Land Value Survey: Overview, Ames, IA., 2017. 


\begin{tabular}{|c|c|c|}
\hline$C_{t-1: t-x}$ & Mean total amount of land allocated to conservation during the previous $X$ years & Hectares \\
\hline$D_{t-1}$ & Previous year's conservation land decision & Hectares \\
\hline$\delta C_{\text {futures: }: Y}$ & Conservation decision based on crop price projections for $Y$ years into the future & Hectares \\
\hline$\delta C_{\text {profit:X }}$ & Conservation decision based on mean past profit of previous $\mathrm{X}$ years & Hectares \\
\hline$\delta \mathrm{C}_{\text {cons }}$ & Conservation decision based on conservation goal & Hectares \\
\hline$C_{\text {neighbor }}$ & Weighted mean conservation land of the farmer agent's neighbors & Hectares \\
\hline Profit $_{\text {diff }}$ & Differences in profit between an acre of crop and an acre of conservation land & $(\$ /$ Hectare $)$ \\
\hline Hectares tot & Total land owned by farmer agent & Hectares \\
\hline Profit $_{\text {crop:t }}$ & Profit derived from an acre of crop land in year $t$ & (\$/Hectare) \\
\hline Profit $_{\text {cons:t }}$ & Profit derived from an acre of conservation land in year $t$ & $(\$ /$ Hectare $)$ \\
\hline CropPrice $_{t}$ & Crop price for yeart & (\$/MT) \\
\hline Yield $t_{t}$ & Average farm yield per hectare for year $t$ & (MT/Hectare) \\
\hline ProdCost $_{t}$ & Production cost per hectare for year $t$ & $(\$ /$ Hectare $)$ \\
\hline FedSub $_{t}$ & Federal subsidy per hectare for year $t$ & (\$/Hectare) \\
\hline Croplns $_{t}$ & Crop insurance per hectare for yeart & $(\$ /$ Hectare $)$ \\
\hline ConsSubsidy & Conservation subsidy rate per hectare for year $t$ & $(\$ /$ Hectare $)$ \\
\hline Cost $_{\text {prairie:t }}$ & Cost of establishing and maintaining native prairie for year $t$ & (\$/Hectare) \\
\hline $\mathrm{P}_{\text {upper }}$ & Upper percentile of historical profit differences & $(\$)$ \\
\hline$P_{\text {middle }}$ & Middle percentile of historical profit differences & (\$) \\
\hline $\mathrm{P}_{\text {lower }}$ & Lower percentile of historical profit differences & $(\$)$ \\
\hline maxChange & Conservation change as a fraction of Cons $_{\max }$ & Dimensionless \\
\hline
\end{tabular}

Table S1. Variables in farmer agent equations.

\begin{tabular}{|c|c|c|}
\hline City Agent Variables & Description & Unit \\
\hline FDam & Current year's flood damage & $(\$)$ \\
\hline FDmax & Maximum attainable flood damage & $(\$)$ \\
\hline Q50 & Discharge at with flood damage if $50 \%$ of maximum & $\left(\mathrm{m}^{3} / \mathrm{s}\right)$ \\
\hline$Q_{\min }$ & Discharge at with flood damage if $1 \%$ of maximum & $\left(\mathrm{m}^{3} / \mathrm{s}\right)$ \\
\hline $\mathrm{Q}_{\max }$ & Discharge at with flood damage if $99 \%$ of maximum & $\left(\mathrm{m}^{3} / \mathrm{s}\right)$ \\
\hline$d Q$ & Width of transition of flood damage curve & $\left(\mathrm{m}^{3} / \mathrm{s}\right)$ \\
\hline $\mathrm{G}_{\mathrm{t}}$ & Government agent conservation goal for the current year $t$ & Hectares \\
\hline $\mathrm{G}_{\mathrm{t}-1}$ & Unfullfilled conservation land from the previous year's $t-1$ conservation goal & Hectares \\
\hline$A_{\text {tot }}$ & Total agricultural land in watershed & Hectares \\
\hline $\mathrm{C}_{\text {tot }}$ & Total land currently in conservation & Hectares \\
\hline $\mathrm{P}$ & Total conservation land to be added to the goal as a percentage of production land & Dimensionless \\
\hline$P_{\text {new }}$ & Variable describing change in conservation goal with flood damage & $(1 / \$)$ \\
\hline
\end{tabular}

Table S2. Variables in city agent equations. 